\title{
Identifying Geometrical Parameters of Fog Lamp and Cornering Lamp, in Compliance with Photometric Requirements
}

\author{
Hatice Özbek, Caner Çil, Ahmet Rodoplu \\ TOFAŞ Turkish Automobile Company, Bursa, Turkey \\ H. Özbek (0000-0002-8271-284X), C. Çil (0000-0003-0164-4636), A. Rodoplu (0000-0002-2764-0978)
}

\begin{abstract}
Automotive lighting, especially the external lighting functions, must have been in line with the international rules. It is essential that lighting functions should have been designed in compliance with essential mechanical, optical, geometric and structural criteria so that the same could be used on the vehicle. In this study, fog lamps, which are used to improve lighting especially in cases of fog, snowfall, heavy rain conditions are discussed for the safety of driver and the passengers. With fog lamps in recent years, cornering function has also been combined in the same equipment. In the current study, geometrical parameters were got for the suitability of fog lamp and cornering lamp on the vehicle. In this way, a method was obtained in order to reveal a product in compliance with legal requirements just in the beginning of design development phase of all vehicles. Due to this method, contribution was made to the design of other parts around the fog lamp so that will not be affected the field of view for illumination.
\end{abstract}

Key Words: Fog Lamp, Cornering, Automotive, Applying Fog Lamp

\section{INTRODUCTION}

During the recent years, automotive industry is a sector that has been progressed. Besides, it a substantial part of the world and Turkish economy. High safety, remarkable style and optimum cost are expected at vehicles by end users. In this case, external lightings are very important. Conformity reports and type approvals for any countries where the vehicles will be sold must be obtained by automobile lighting manufacturers. It is essential that the conformity and design of the position of the lamps on the vehicles must comply with the regulations. In the study conducted in this respect, a method was developed in which front fog lamps and fog lamps with cornering would fulfil proper legal requirements on the vehicle. It was also addressed how geometrical and optical parameters of front fog lamps and fog lamps with cornering direct the style studies, which is the first stage that determined the visual structure of the vehicle in automotive sector.

\subsection{Fog Lamp and Cornerıng Lamp}

Front fog lamp for automobile is an important guarantee for safe driving in fog or rain and other low visibility driving conditions [1]. Front fog lamps ensure that the road, lanes on the road, traffic signs, pedestrians etc. can be seen on foggy and rainy weathers. Owing to fog lamps, it becomes easy to see the road and the objects around the road. Therefore, the accident risk to be encountered because of vision is minimized. Front fog lamps can be utilized in any vehicle categories (Figure: 1). There are 2 fog lamps on the vehicle and the light, given out is either white or selective yellow [2].

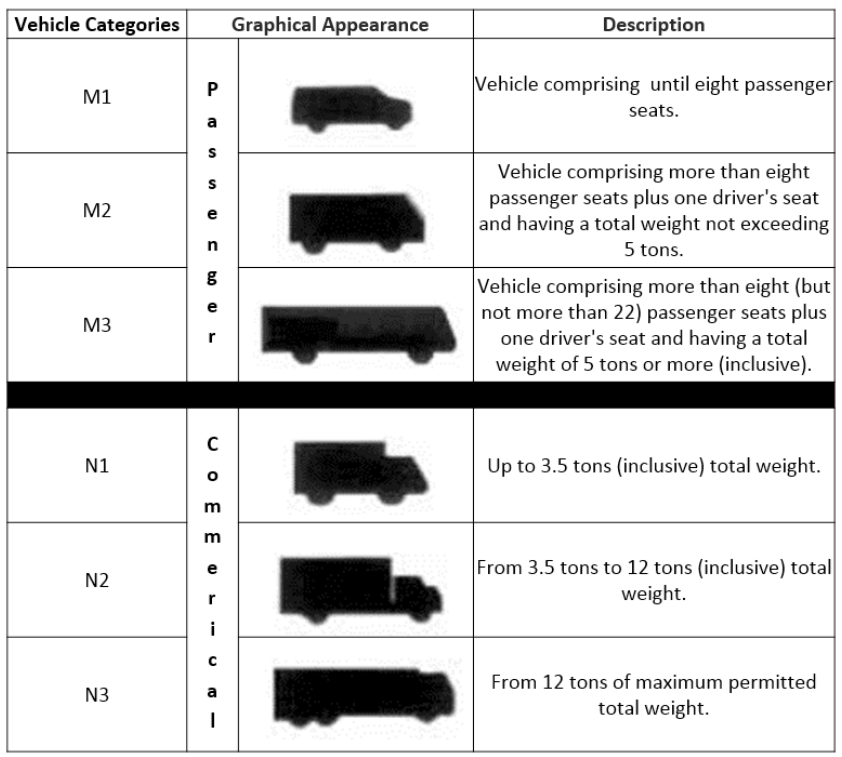

Figure 1: Vehicle Categories.
${ }^{*}$ Corresponding authour

Email: haticeozbek1688@gmail.com
European Mechanical Science, March 2019; 3(1): 32-36 doi: https://doi.org/10.26701/ems.450236

Received: August 1, 2018

Accepted: November 23, 2018 
Cornering lamp" means a lamp used to provide supplementary illumination of that part of the road which is located near the forward corner of the vehicle at the side towards which the vehicle is going to turn [3].

\subsubsection{Legal Requirements Regarding Fog Lamp and Cor- nering Lamp}

Front fog lamp must have characteristics which ensure safe driving in rainy, foggy and similar weather conditions that restrict vision. In the regulation by United Nations European Economy Commission (ECE 19), front fog lamp requirements are addressed in detail. Photometric requirements of front fog lamp must comply with F3 category. F3 category was designed in order to increase the photometric performance and ensure a safer driving [4]. Photometric requirements of front fog lamp in accordance with ECE19 are indicated in Figure 2. The lighting zone that fall upon a screen at a distance of 25 meters is as stated in Figure 2. Luminous intensities are different for per zones (Table: 1 ).

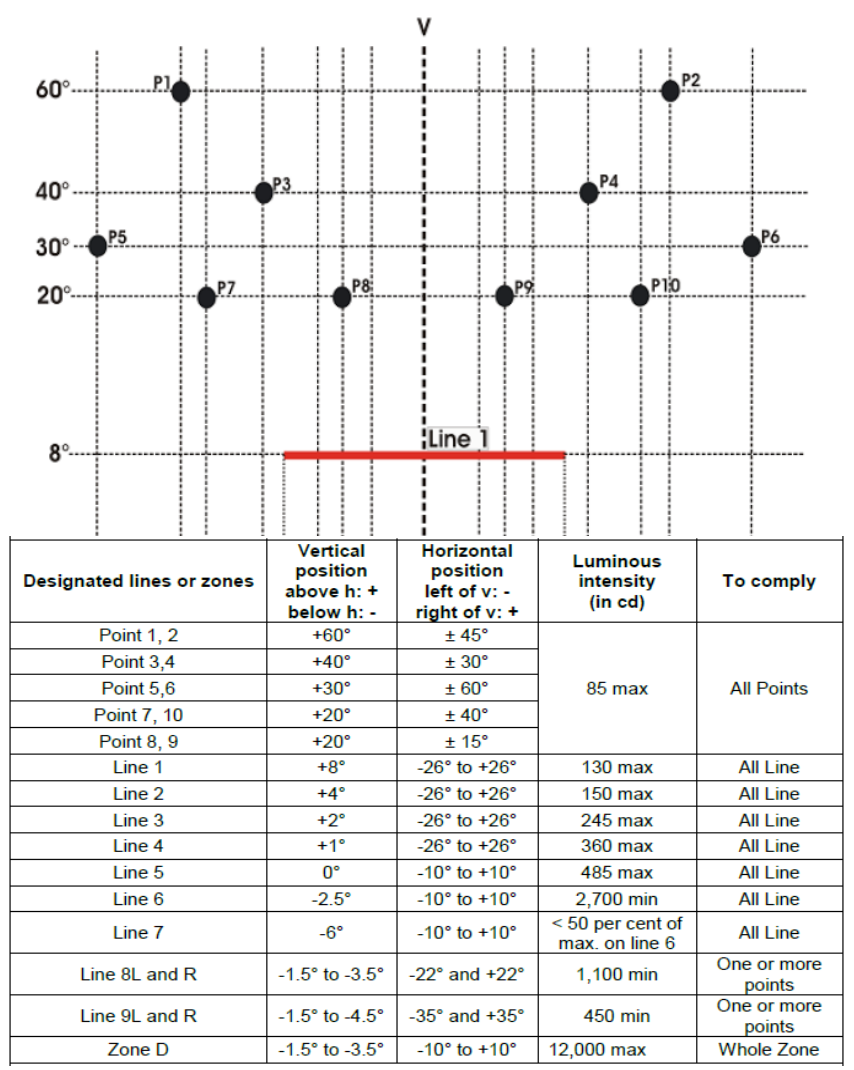

All necessities must be ensured for have been certification by the product. Also, photometric requirements of the cornering lamp are discussed in detail in ECE 119. Essential luminous zones and lighting intensities are presented in $\mathrm{Fi}$ gure 3 .

\subsubsection{The Position of Fog Lamp and Cornering Lamp on The Vehicle And Their Compliance With Photometric An- gle of Sight}

In order that front fog lamp could receive vehicle homologation, it must be located on the vehicle under the limit conditions, specified in ECE 19; and cornering must be located under the limit conditions, specified in ECE 119 (Figure: 4).
Besides, in ECE 48, is the general external lighting regulation, is referred to the front fog lamp and cornering lamps positions on the car. The presence of partitions or other items of equipment near the front fog lamp shall not give rise to secondary effects causing discomfort to other road users [2].

\section{$\mathbf{L}$}

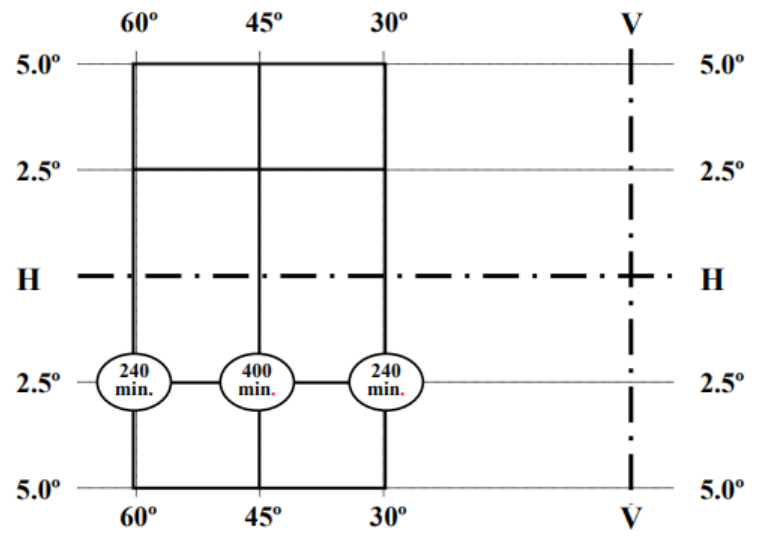

L

Figure 3: Photometric area and luminous intensity requirements of cornering lamp.

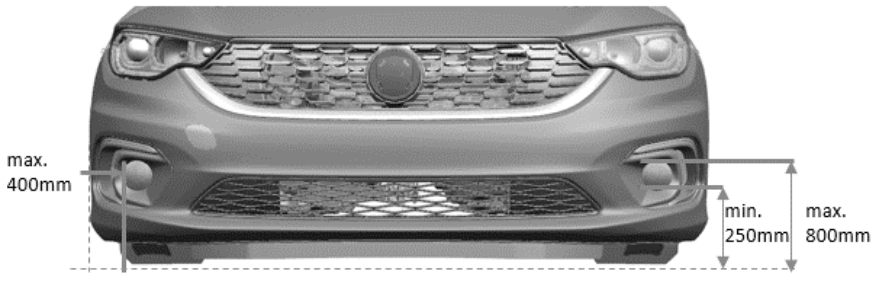

Figure 4: Geometrical position of fog lamp and cornering
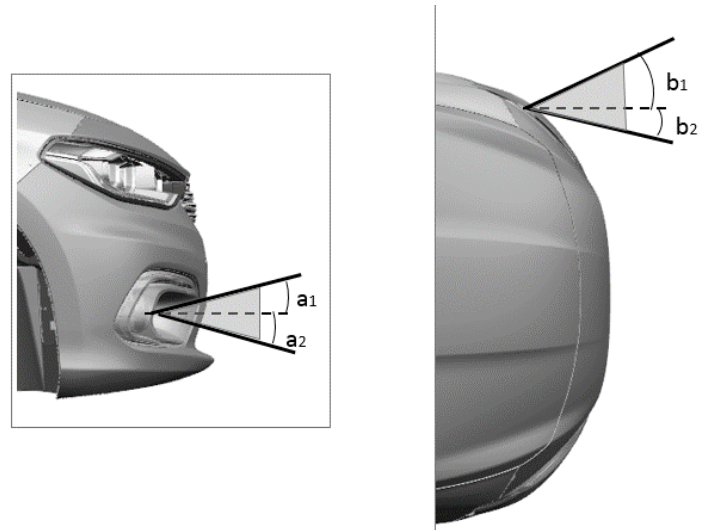

Figure 5: Photometric visibility angles of fog lamp.

However, there shouldn't be any component in front of the fog lamp and cornering that will block photometric visibility angles. Visibility angles of fog lamp and cornering are indicated in Figure 5, Figure 6 and Table2, Table3.

Table 2: Photometric visibility angles of fog lamp.

\begin{tabular}{|c|c|c|c|}
\hline a1 & a2 & b1 & b2 \\
\hline upwards & downwards & outwards & inwards \\
\hline $5^{\circ}$ & $5^{\circ}$ & $45^{\circ}$ & $10^{\circ}$ \\
\hline
\end{tabular}

Table 3: Photometric visibility angles of cornering.

\begin{tabular}{|c|c|c|c|}
\hline a'1 & a'2 & $b^{\prime} 1$ & $b^{\prime} 2$ \\
\hline upwards & downwards & outwards & outwards \\
\hline $10^{\circ}$ & $10^{\circ}$ & $60^{\circ}$ & $30^{\circ}$ \\
\hline
\end{tabular}



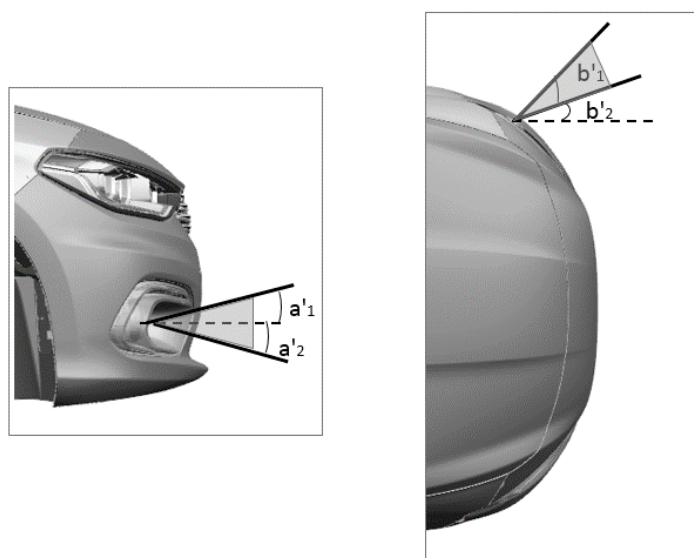

Figure 6: Photometric visibility angles of cornering.

\subsection{Surrounding Parts of Fog Lamp and Cornering Lamp}

While the fog lamp is designed, limit conditions must be identified in consideration of surrounding parts. The geometry of the bumper and the fog lamp frame around the fog lamp must in no sense be within the photometric visibility angles, specified in part 1.1.2. In cases where they coincide with the visibility angles, the vehicle is not allowed to obtain homologation and used under serial conditions.

Fog lamp frame: This product that was designed in order to facilitate the assembly of the fog lamp on the vehicle, is protected the fog lamp against the potential impacts and is used for visual purposes (Figure: 7).

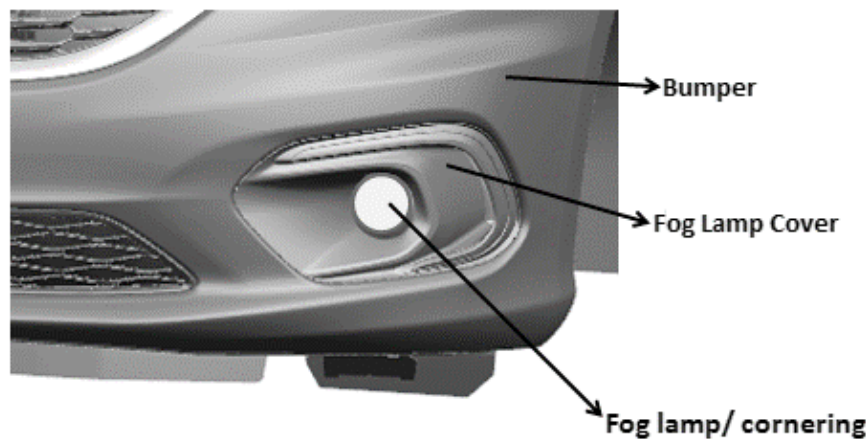

Figure 7: Surrounding parts of fog lamp and cornering.

Bumper: Fog lamp is generally located on the front bumper. There are also some vehicles that is integrated the Front Fog in the Main headlamp. Bumper absorbs the accidental kinetic energy by deflection in low-speed impact and by defor- mation in high-speed impact [5]. (Figure:7).

\section{MATERIALS AND METHODS}

There are 2 main terms was defined regarding the use of the fog lamp and cornering lamp on the vehicle. First, the product must, on its own, will be designed and manufactured in compliance with the rules, set forth in ECE 48, ECE 19 and ECE 119 and obtain a homologation certificate; and secondly; front fog lamp and cornering lamp must be positioned defined area on the vehicle and a vehicle homologation must be received.

Vehicle design is changed constantly with customer request, competitiveness and new technologies. Style life of a vehicle put on the market is 8 years on average. At the end of these 8 years, it is inevitable to put a vehicle on the market with a new design and features for competitiveness. These 8 years also include the slight changes made once in every 2 or 4 years for offering changed products with more slight modifications to the customers. With these modifications; lighting functions should once again be controlled and conformity (homologation) certificates that meet regulations must be obtained [6].

In the current study, parameters were identified for proper assembly of a standard fog lamp on the vehicle. Thus, a method was obtained for use of the fog lamp a cornering that received homologation independent of the model of the vehicle.

\section{Step 1:}

In the technical drawing for the fog lamp and cornering to be utilized on the vehicle, minimum visibility zones that meet the photometric values in ECE regulation are identified. The work was initiated in consideration of such information. (Figure 8)

\section{Step 2:}

3D data of the fog lamp was opened with proper 3D design program. A lens surface was extracted from the lens of the fog lamp (Figure: 9)

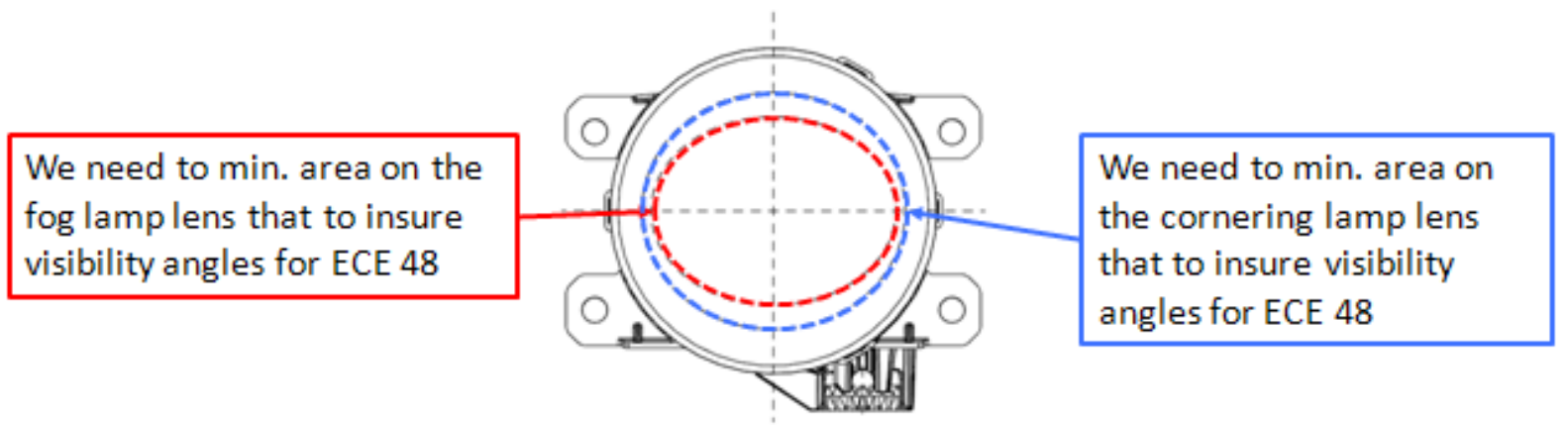

Figure 8: Minimum visibility zones according to ECE regulation. 

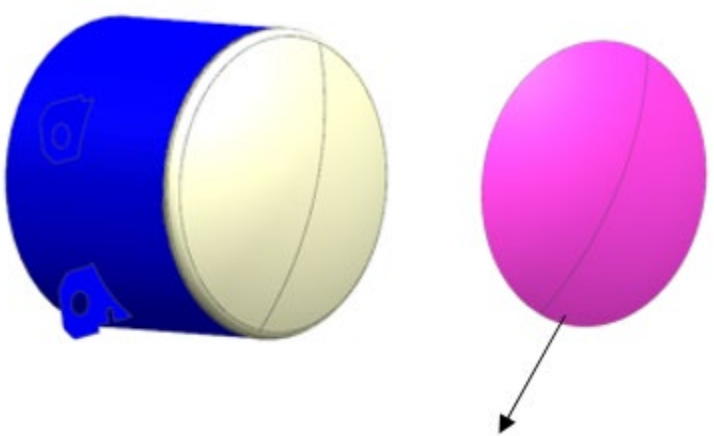

Extract lens surface

Figure 9: Extracted lens surface.

Step 3:

A datum plane was created on the centre of extracted lens surface and ellipse was drawn to measure of min. insure visibility angles according to fog lamp/cornering technical drawing (or bigger than this measure). The surface remaining outside this area was trimmed (Figure 10).
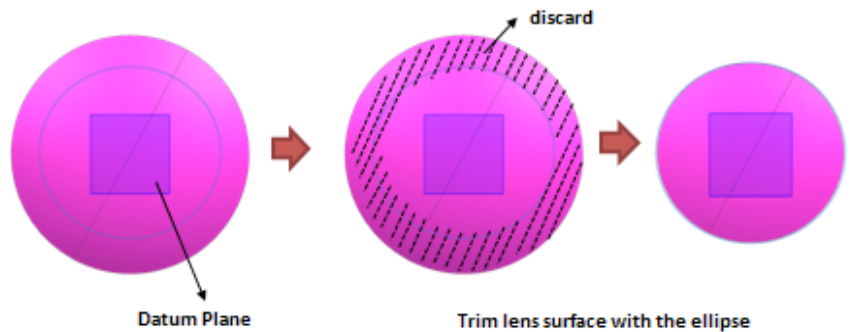

Figure 10: Trimmed lens surface according to technical drawing.

Step 4:

Trimmed lens surface is cut individually in the middle on $Y$ axis and $\mathrm{Z}$ axis. A reference normal line is drawn from the centre of the lens cut on $\mathrm{Y}$ axis. In order to determine the photometric visibility zone, set forth in ECE 48 for the fog lamp without cornering; 5'-degree virtual lines are created towards the vehicle up-downwards angle as in Figure 11. Likewise, a reference normal line is drawn from the centre of the lens cut on $\mathrm{Z}$ axis. Virtual lines are created so as to be 10 '-degree towards the vehicle inwards and $45^{\prime}$-degree towards the vehicle outwards (Figure 11).

If cornering lamp feature is studied; it will be required to adapt the virtual lines with the angles, specified in Table3 with the virtual lines created for the fog lamp.

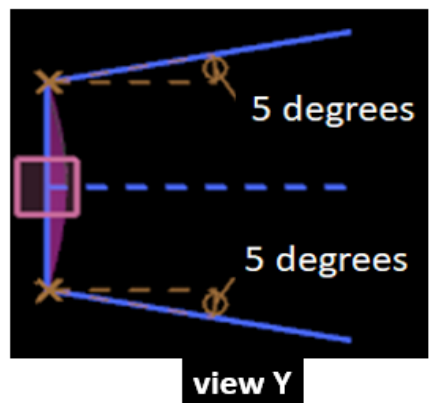

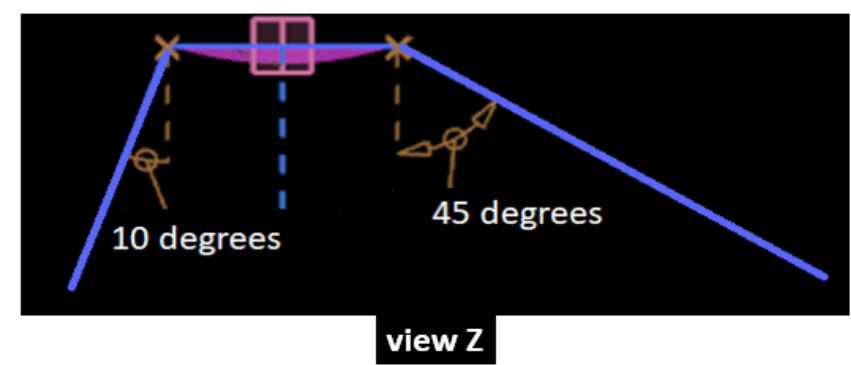

Figure 11: Photometric visibility angles.

Step 5:

Using the virtual lines and the lens surface, created on $Y$ and Z-axis, a conical geometry in compliance with the photometric limit values, was achieved as in Figure 12.
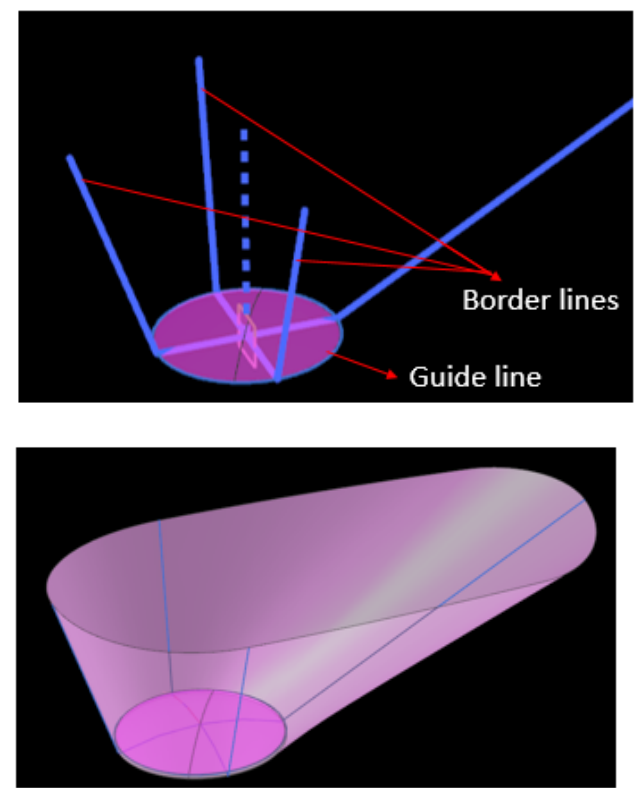

Figure 12: Conical geometry for compliance with the photometric limit values.

\section{RESULT AND DISCUSSIONS}

The fog lamp cone, which was obtained five steps, is located in the area of fog lamp on the vehicle that the fog lamp is desired to be used (Figure 13). The expectation was that it made no interference with the surrounding parts on car. In this respect, the design of the fog lamp frame and bumper is carried out. Also with this method was ensured input to knowledge base engineering. Knowledge base engineering with fog lamp cone is determined boundary conditions for design of bumper and fog lamp cone and others surrounding parts. If any problem is seen when beginning of the design, this method is interfered to the problem easily.

This study's another result is it is given direction to the new bumper style. The method that enables the design of the surrounding parts of the fog lamp; it was developed - in the beginning of the design phase- to prevent any regulative problems likely to take place in the future. 


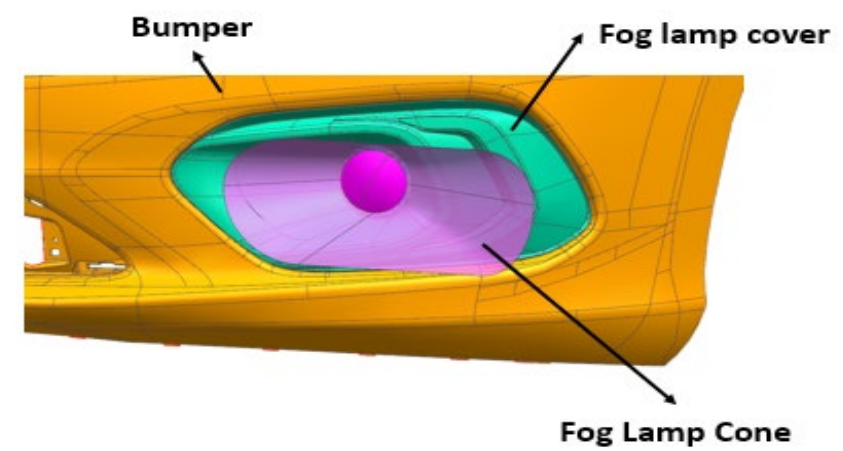

Lighting Conference.

Figure13: Controlling visibility with fog lamp cone.

Accordingly, true designs are obtained and it is gained engineering time with method of the Identifying Geometrical Parameters of Fog Lamp and Cornering Lamp, in Compliance with Photometric Requirements.

\section{CONCLUSIONS}

Certain criteria and legal requirements are taken into consideration while a vehicle is in design phase. In order to prevent the problems likely to be encountered in future, one of the most important criteria is to start the design on the basis of the regulations and norms with international validity.

In the current study, parameters were determined regarding the proper settlement of a standard fog lamp on a vehicle. A fog lamp conic was created in compliance with these parameters. Owing to the fog lamp conic method, achieved in this respect, essential limit values were determined so that the fog lamp and cornering would be used on the vehicle. Thus, a method that will enable the design of the surrounding components of the fog lamp was obtained.

This method was developed for to prevent avoid of noncompliance with legal requirements likely to take place in the future, when the beginning of the vehicle design.

This method will ensure that a fast and reliable control in vehicle development phase as it can be applied to all fog lamps and cornering equipment.

\section{REFERENCES}

[1] W. Hong, L. Xiufeng and G. Peng (2017). Design of an optical lens combined with a total internal reflection (TIR) free form surface for a LED front fog lamp. Optics \& Laser Technology 88: 11-16

[2] United Nations Economic Commission for Europe Vehicle Regulations (UNECE), Regulation No. 48 - Rev.12

[3] United Nations Economic Commission for Europe Vehicle Regulations (UNECE), Regulation No. 119 - Rev.2

[4] United Nations Economic Commission for Europe Vehicle Regulations (UNECE), Regulation No. 19 - Rev.7

[5] M.M. Davoodi, S.M. Sapuan, A. Aidy, N.A. Abu Osman, A.A. Oshkour, W.A.B. Wan Abas (2012). Development process of new bumper beam for passenger car: A review. Materials and Design 40: 304-313

[6] H. Ozbek, C. Cil, A. Rodoplu, S. Dogru (2017). Ground clearance control and its importance of automotive lighting functions with respect to changes in weight and loads of vehicles. 11th National 